\title{
Approximate ESPs on Surfaces of Polytopes Using a Rubberband Algorithm
}

\author{
Fajie $\mathrm{Li}^{1}$, Reinhard Klette ${ }^{2}$, and $\mathrm{Xue} \mathrm{Fu}^{3,4}$ \\ ${ }^{1}$ Institute for Mathematics and Computing Science, University of Groningen \\ P.O. Box 800, 9700 AV Groningen, The Netherlands \\ ${ }^{2}$ Computer Science Department, The University of Auckland \\ Private Bag 92019, Auckland 1142, New Zealand \\ ${ }^{3}$ Faculty of Economics, University of Groningen \\ P.O. Box 800, 9700 AV Groningen, The Netherlands \\ ${ }^{4}$ School of Public Finance, Jiangxi University of Finance and Economy \\ Nanchang, 330013, China
}

\begin{abstract}
Let $p$ and $q$ be two points on the surface of a polytope $\Pi$. This paper provides a rubberband algorithm for computing a Euclidean shortest path between $p$ and $q$ (a so-called surface ESP) that is contained on the surface of $\Pi$. The algorithm has $\kappa_{1}(\varepsilon) \cdot \kappa_{2}(\varepsilon) \cdot \mathcal{O}\left(n^{2}\right)$ time complexity, where $n$ is the number of vertices of $\Pi, \kappa_{i}(\varepsilon)=\left(L_{0 i}-L_{i}\right) / \varepsilon$, for the true length $L_{i}$ of some shortest path with initial (polygonal path) length $L_{0 i}$ (used when approximating this shortest path), for $i=1,2$. Rubberband algorithms follow a straightforward design strategy, and the proposed algorithm is easy to implement and thus of importance for applications, for example, when analyzing 3D objects in 3D image analysis, such as in biomedical or industrial image analysis, using 3D image scanners.
\end{abstract}

Keywords: Rubberband algorithm, Euclidean shortest path, surface ESP.

\section{Introduction}

Let $\Pi$ be a connected polyhedral domain such that its frontier is a union of a finite number of triangles. An obstacle is a connected, bounded polyhedral component in the complement $\mathbb{R}^{3} \backslash \Pi$ of $\Pi$. Let $p, q \in \Pi$ such that $p \neq q$. The general Euclidean shortest-path problem (ESP) asks to find a shortest polygonal path $\rho(p, q)$ which is either completely contained in $\Pi$, or just not intersecting any (topologic) interior of a finite number of given obstacles.

This problem is actually a special case of the problem of planning optimal collision-free paths for a robot system; for its specification and a first result, see 1]. This paper presented in 1984 a doubly exponential time algorithm for solving the general obstacle avoidance problem. 2] improved this by providing a singly exponential time algorithm. The result was further improved by a PSPACE algorithm in 3. Since the general ESP problem is known to be NP-hard 4, special cases of the problem have been studied afterwards. 5] gave a polynomial time algorithm for ESP calculations for cases where all obstacles are convex and

D. Mery and L. Rueda (Eds.): PSIVT 2007, LNCS 4872, pp. 236-247 2007.

(C) Springer-Verlag Berlin Heidelberg 2007 
the number of obstacles is small. 6] solved the ESP problem with an $\mathcal{O}\left(n^{6 k-1}\right)$ algorithm assuming that all obstacles are vertical "buildings" with $k$ different values for height.

[1] is the first publication considering the special case that the shortest polygonal path $\rho(p, q)$ is constrained to stay on the surface of $\Pi$. [1] presented an $\mathcal{O}\left(n^{3} \log n\right)$ algorithm where $\Pi$ was assumed to be convex. [7 improved this result by providing an $\mathcal{O}\left(n^{2} \log n\right)$ algorithm for the surface of any bounded polyhedral $\Pi$. The time complexity was even reduced to $\mathcal{O}\left(n^{2}\right)$ [8]. So far, the best known result for the surface ESP problem is due to [9]; it improved in 1999 the time complexity to $\mathcal{O}\left(n \log ^{2} n\right)$, assuming that there are $\mathcal{O}(n)$ vertices and edges on $\Pi$.

This paper provides a rubberband algorithm (RBA) for computing approximate surface ESP. The algorithm has

$$
\kappa_{1}(\varepsilon) \cdot \kappa_{2}(\varepsilon) \cdot \mathcal{O}\left(n^{2}\right)
$$

time complexity, where $n$ is the number of vertices of $\Pi$, and

$$
\kappa_{i}(\varepsilon)=\frac{L_{0 i}-L_{i}}{\varepsilon}
$$

for the true length $L_{i}$ of some kind of shortest path with length $L_{0 i}$ of the used initial polygonal path, for $i=1,2$.

Although this rubberband algorithm is not the most efficient, it follows a straightforward design strategy, and the proposed algorithm is easy to implement. (See [10] for results on implementing rubberband algorithms for various shortest path problems.)

We generalize a rubberband algorithm from solving the 2D ESP of a simple polygon (see 11] for this 2D algorithm) to a solution for the surface ESP of polytopes. Considering the difficulty of the general ESP problem, our approach is very important for applications, e.g. when analyzing $3 \mathrm{D}$ objects in $3 \mathrm{D}$ image analysis (such as in biomedical or industrial image analysis, using 3D image scanners). For shortest paths on digital surfaces (in the context of 3D picture analysis), also known as geodesics, see the monograph 12. One of the earlier publications, related to the calculation of surface geodesics, is [13].

The rest of this paper is organized as follows: Section 2 provides the definitions of some useful notions. Section 3 presents four procedures being subroutines of the Main Algorithm. Section 4 proves the correctness of the Main Algorithm. Section 5 analyses the time complexities for involved procedures and Main Algorithm. Section [6 illustrates the main ideas behind the steps of the Main Algorithm by a simple example. Section 7 summarizes the paper.

\section{Definitions}

Let $\Pi$ be a polytope (see Figure 1 for an example). Let $T=\left\{\triangle_{1}, \triangle_{2}, \ldots, \triangle_{m}\right\}$ be a set of triangles such that $\partial \Pi=\cup_{i=1}^{m} \triangle_{i}$ and $\triangle_{i} \cap \triangle_{j}=\emptyset$ or $=e_{i j}$ or $=v_{i j}$, 


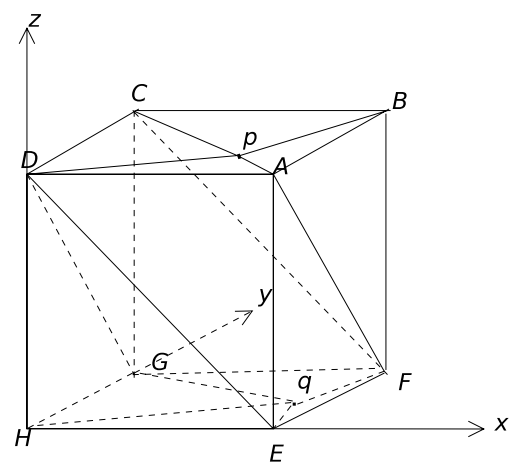

Fig. 1. An unit cube within the $x y z$-coordinate system, where $p=(0.76,0.12,1)$, $q=(0.9,0.24,0)$

where $e_{i j}\left(v_{i j}\right)$ is an edge (vertex) of both $\triangle_{i}$ and $\triangle_{j}, i \neq j$, respectively, with $i, j=1,2, \ldots, m$.

We construct a corresponding simple graph $G_{\Pi}=\left[V_{\Pi}, E_{\Pi}\right]$ where $V_{\Pi}=$ $\left\{v_{1}, v_{2}, \ldots, v_{m}\right\}$. Each $v_{i}$ is a triangle. Edges $e \in E_{\Pi}$ are defined as follows: If $\triangle_{i} \cap \triangle_{j}=e_{i j} \neq \emptyset$, then we have an edge $e=v_{i} v_{j}$ (where $e_{i j}$ is an edge of both triangles $\triangle_{i}$ and $\triangle_{j}$ ); and if $\triangle_{i} \cap \triangle_{j}=\emptyset$ or a vertex, then there is not an edge between $v_{i}$ and $v_{j}, i<j$ and $i, j=1,2, \ldots, m$.

In such a case we say that $G_{\Pi}$ is a corresponding graph with respect to the triangulated polytope $\Pi$. See Figure 2 for an example. Analogously, we can define a corresponding graph for a connected surface segment (a subsurface) of a polytope. Abbreviated, we may also speak about "the graph for a polytope" or "the graph for a subsegment of a surface".

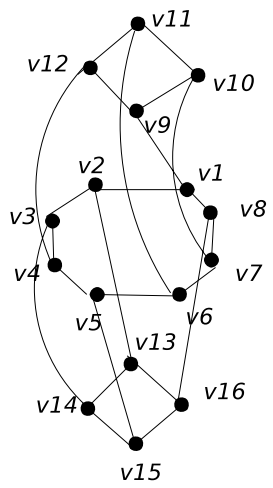

Fig. 2. A (3-regular) corresponding graph of the polytope in Figure 1 
A triangulated polytope $\Pi$ can also be thought as being a graph such that each vertex of $\Pi$ is a vertex of this graph, and each edge of a triangle is an edge of this graph. We denote this graph by $G_{\Pi}^{\prime}$.

Let $p \neq q, p, q \in V\left(G_{\Pi}^{\prime}\right)$; if $\rho$ is a cycle of $G_{\Pi}^{\prime}$ such that $G_{\Pi}^{\prime} \backslash \rho$ has two components, denoted by $G_{1}$ and $G_{2}$ with $p \in V\left(G_{1}\right)$ and $q \in V\left(G_{2}\right)$, then $\rho$ is called a cut cycle of $G_{\Pi}^{\prime}$ or $\Pi$. For example, in Figure 1, $A B C D A$ or $A F G D A$ are cut cycles of $\Pi$.

An approximate cycle is a graph such that it consists of a cycle plus a few more vertices, each of which is of degree one only, and (thus) adjacent to a vertex on the cycle. (The graph later shown in Figure 4 is an approximate cycle.)

A band is a subsurface of a polytope $\Pi$ such that the corresponding graph of it is a cycle or an approximate cycle.

A band can also be thought as being a subgraph of $G_{\Pi}^{\prime}$. Let $E^{\prime}$ be the subset of all the edges of a triangulated band such that each edge belongs to a unique triangle. Then $E^{\prime}$ consists of two cycles. Each of them is called a frontier of the band. For example, in Figure 1] $A B C D A$ and $E F G H E$ are two frontiers of a band whose triangles are perpendicular to the $x o y$-plane.

If two triangulated bands share a common frontier, then they are called continuous (in the sense of "continuation").

\section{Algorithms}

Without loss of generality, we can assume that $p \neq q, p$ and $q \in V(\Pi)$.

\subsection{Separation}

The following procedure finds a cut cycle to separate $p$ and $q$ such that either $p$ or $q$ is not a vertex of the cut cycle. (This procedure will be used in Step 1 of the Main Algorithm below.)

\section{Procedure 1}

Input: $G_{\Pi}^{\prime}=[V(\Pi), E(\Pi)]$, and two vertices $p \neq q \in V(\Pi)$.

Output: The set of all vertices of a cycle $\rho$ in $G$ such that, if we cut the surface of $\Pi$ along $\rho$ into two separated parts, then $p$ and $q$ are on different parts respectively.

1. Let $N_{p}=\{v: v p \in E(\Pi)\}$ (i.e., the set of all neighbors of $p$ ).

2. Select $u, v \in N_{p}$ such that $\angle u p v \neq 180^{\circ}$. In other words, $u v \in E(\Pi)$.

3. Let $V=\{p, v\}$.

4. Let $N_{v}=\left\{w^{\prime}: w^{\prime} v \in E(\Pi)\right\}$ (i.e., the set of all neighbors of $v$ ).

5. Take a vertex $w \in N_{v} \backslash V$.

6. If $w=u$, then stop. Otherwise, let $V=V \cup\{w\}, v=w$ and go to Step 4 .

7. If $q \notin V$, then output $V$. Otherwise, output $V \backslash\{q\}$. 
For example, in Figure 1] $\rho$ can be either $A B C D A$ or $A F G D A$, but it can not be $A E H D A$.

\subsection{Step Set Calculation}

The following procedure computes step bands (i.e., the step set for the second level RBA). It will be used in Step 2 of the Main Algorithm below.

\section{Procedure 2}

Input: $G_{\Pi}^{\prime}=[V(\Pi), E(\Pi)]$, and $\rho$ : the cut cycle obtained with Procedure 1. Without loss of generality, we can assume that $p \in V(\rho)$ and $q \notin V(\rho)$.

Output: The set of the step bands $S=\left\{B_{1}, B_{2}, \ldots, B_{m}\right\}$ such that $p \in V\left(B_{1}\right)$ and $q \in V\left(B_{m}\right)$.

1. Let $S=\emptyset, \Pi_{1}=\Pi$ and $\rho_{1}=\rho$.

2. While $q \notin V\left(\rho_{1}\right)$, do the following:

2.1. Let $\Pi_{2}=\Pi_{1}-\rho_{1}$ such that $q \in V\left(\Pi_{2}\right)$. (Note: the used "minus" in graph theory can also be written as $\Pi_{1} \backslash \rho_{1}$; in other words, we delete each vertex in $\rho_{1}$ and each edge of $\Pi_{1}$ which is incident with a vertex of $\rho_{1}$.)

2.2. Let $\rho_{2}$ be the frontier of $\Pi_{2}$.

2.3. Let $\Pi_{1}, \rho_{1}$ and $\rho_{2}$ as the input, compute a band $B=G_{\Pi_{1}}\left(V\left(\rho_{1}\right) \cup V\left(\rho_{2}\right)\right)$ (the induced subgraph of $G_{\Pi_{1}}$ ).

2.4. Update $\rho_{1}$ and $\Pi_{1}$ by letting $\rho_{1}=\rho_{2}$ and $\Pi_{1}=\Pi_{2}$.

2.5. Let $S=S \cup\{B\}$.

3. Output $S$.

For example, in Figure 1 if a single vertex can be thought of as being a band, then we can have $S=\left\{B_{1}, B_{2}, B_{3}\right\}$, where $B_{1}=p, B_{2}$ is the band such that $V\left(B_{2}\right)=\{A, B, C, D, E, F, G, H\}$, and $B_{3}=q$.

\subsection{Step Segments in a Single Band}

The following procedure computes step segments in a single band (i.e., a subset of the step set for the initialization of the RBA). (It will be used in Step 1.1 of Procedure 4 below.)

\section{Procedure 3}

Input: The triangulated band $B$ and two vertices $u, v \in V(B)$ such that $u$ and $v$ are on two different frontiers of $B$, denoted by $\rho_{1}$ and $\rho_{2}$ (i.e., $u \in V\left(\rho_{1}\right)$ and $v \in V\left(\rho_{2}\right)$.

Output: Two step sets of segments (edges) $S_{1}$ and $S_{2}$ such that either $S_{1}$ or $S_{2}$ contains the vertices of a surface $E S P$ of $B$ from $u$ to $v$.

Let $\triangle_{u}, \triangle_{v}$ be the triangles such that $u \in \partial \triangle_{u}$ and $v \in \partial \triangle_{v}$, respectively. Let $w_{u}$ and $w_{v} \in V\left(G_{B}\right)$ such that $w_{u}$ and $w_{v}$ correspond to $\triangle_{u}$ and $\triangle_{v}$, respectively. 


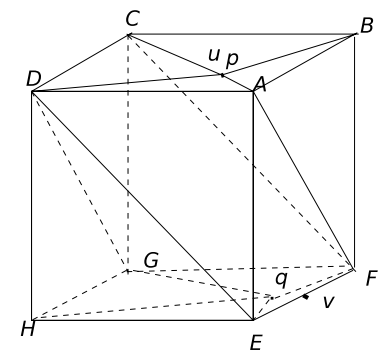

Fig. 3. A unit cube such that $u=p$, and $v$ is the center of $E F$

By the definition of a band (see Section 2), there is a cycle, denoted by $\rho_{B}$, such that either $w_{u}$ (respectively, $\left.w_{v}\right) \in V\left(\rho_{B}\right)$ or the unique neighbor of $w_{u}$ (respectively, $\left.w_{v}\right)$ is in $V\left(\rho_{B}\right)$.

For example, in Figure 3, the frontier of $B$ consists of two cycles $u A B C D u$ and $E F G H E$. We have that $\triangle_{u}=\triangle p D A, \triangle_{v}=\triangle A E F . S_{1}=\{A D, A E\}$ and $S_{2}=\{D A, D E, D H, D G, C G, C F, B F, A F\}$.

Case 1: Both $w_{u}$ and $w_{v}$ are in $V\left(\rho_{B}\right)$. In this case, $\rho_{B}$ can be decomposed into two paths from $w_{u}$ and $w_{v}$, denoted by $P_{1}$ and $P_{2}$. Let $\left\{\triangle_{1}, \triangle_{2}, \ldots, \triangle_{m_{1}}\right\}$ be the sequence of triangles corresponding to the sequence of the vertices of $P_{1}$.

Let $\left\{e_{1}, e_{2}, \ldots, e_{m_{1}-1}\right\}$ be a sequence of edges such that $e_{i}=\triangle_{i} \cap \triangle_{i+1}$, where $i=1,2, \ldots, m_{1}-1$.

Let $\left\{e_{1}^{\prime}, e_{2}^{\prime}, \ldots, e_{m_{1}-1}^{\prime}\right\}$ be a sequence of edges such that $e_{i}^{\prime}$ is obtained by removing a sufficiently small segment (Assume that the length of the removed segment equals $\delta^{\prime}$.) from both endpoints of $e_{i}$, where $i=1,2, \ldots, m_{1}-1$.

The set $\left\{e_{1}^{\prime}, e_{2}^{\prime}, \ldots, e_{m_{1}-1}^{\prime}\right\}$ is the approximate step set we are looking for.

Case 2: Both $w_{u}$ and $w_{v}$ are not in $V\left(\rho_{B}\right)$. Again, by the definition of a band (see Section 2), let $w_{u}^{\prime}\left(w_{v}^{\prime}\right)$ be the unique neighbor of $w_{u}\left(w_{v}\right)$ such that $w_{u}^{\prime}$ and $w_{v}^{\prime} \notin V\left(\rho_{B}\right)$.

In this case, $\rho_{B}$ can be decomposed into two paths from $w_{u}^{\prime}$ and $w_{v}^{\prime}$, denoted by $P_{1}^{\prime}$ and $P_{2}^{\prime}$. Appending $w_{u}$ and $w_{v}$ to both ends of $P_{1}^{\prime}$ and $P_{2}^{\prime}$, we obtain

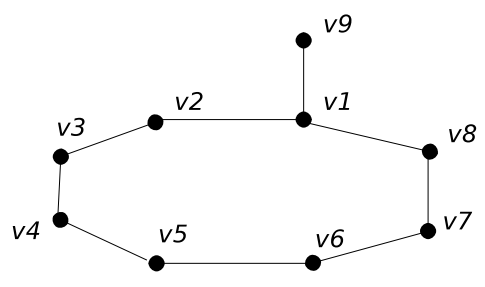

Fig. 4. The corresponding graph with respect to $B$; the two frontiers of $B$ are $p A B C D p$ and $E F G H E$ in Figure $3 v 9$ corresponds to $\triangle p D A$, and $v 2$ corresponds to $\triangle A E F$. 
two paths, denoted by $P_{1}$ and $P_{2}$. Analogous to Case 1 , we can compute the approximate step set.

Case 3: Only one of either $w_{u}$ or $w_{v}$ is not in $V\left(\rho_{B}\right)$. We can compute the approximate step set, analogously to Cases 1 and 2 .

\subsection{Initializations}

The following procedure is the initialization procedure of the RBA. It will be used in Steps 7.2 and 8.2 of the Main Algorithm below.

\section{Procedure 4}

Input: Two continuous triangulated bands $B_{1}$ and $B_{2}$, and three vertices $u_{1}$, $u_{2}$ and $u_{3}$, all three in $V\left(B_{1} \cup B_{2}\right)$, such that $u_{1}$ and $u_{2}$ are on two different frontiers of $B_{1}$, denoted by $\rho_{1}$ and $\rho_{2} ; u_{3}$ is on the frontier denoted by $\rho_{3}\left(\neq \rho_{2}\right)$, of $B_{2}$.

Output: The set of vertices of an approximate ESP on the surface of $B_{1} \cup B_{2}$, from $u_{1}$ to $u_{3}$.

Let $e_{u_{2}} \in E\left(\rho_{2}\right)$ such that $u_{2} \in e_{u_{2}} ; l$ a sufficiently large integer; and $E=$ $E\left(\rho_{2}\right)$.

1. While $E \neq \emptyset$, do the following:

1.1. Let $G_{B_{i}}$ and $u_{i}, u_{i+1}$ be the input; apply Procedure 3 to compute step segments in band $B_{i}$, denoted by $S_{B_{i}}$, where $i=1,2$.

1.2. Let $S_{12}=S_{B_{1}} \cup S_{B_{2}}$ be the input. Apply Algorithm 1 of [1] to compute an approximate ESP on the surface of $B_{1} \cup B_{2}$. This is denoted by $\rho_{e_{u_{2}}}$, and it connects $u_{1}$ with $u_{3} 1$

1.3. Let the length of $\rho_{e_{u_{2}}}$ be equals $l\left(\rho_{e_{u_{2}}}\right)$.

1.4.1. If $l\left(\rho_{e_{u_{2}}}\right)<l$ then let $V=V\left(\rho_{e_{u_{2}}}\right)$.

1.4.2. If $l\left(\rho_{e_{u_{2}}}\right)=l$ then let $V=\min _{l e x i}\left\{V, V\left(\rho_{e_{u_{2}}}\right)\right\}$ (minimum with respect to lexicographic order).

1.5. Let $E=E \backslash\left\{e_{u_{2}}\right\}$.

1.6. Take an edge $e \in E$ and let $u_{2}$ be one endpoint of $e$; let $e_{u_{2}}=e$; go to Step 1.1.

2. Output $V$.

\subsection{The Main Algorithm}

The main algorithm defines now the iteration steps of the RBA.

Input: $G_{\Pi}^{\prime}=[V(\Pi), E(\Pi)]$, and two vertices $p \neq q, p, q \in V(\Pi)$; accuracy constant $\varepsilon$.

${ }^{1}$ Note that Algorithm 1 of [1] still works when the line segments in the step set are in $3 \mathrm{D}$ space. 
Output: The set of vertices of an approximate ESP on the surface of $\Pi$.

1. Let $G_{\Pi}^{\prime}, p$ and $q$ be the input; apply Procedure 1 to compute a cut cycle which separates $p$ and $q$, denoted $\rho_{p q}$.

2. Let $G_{\Pi}^{\prime}$ and $\rho_{p q}$ be the input; apply Procedure 2 to compute step bands $S$ $=\left\{B_{1}, B_{2}, \ldots, B_{m}\right\}$ such that $p \in V\left(B_{1}\right)$ and $q \in V\left(B_{m}\right)$.

3. Let $p_{i}$ be a point on the frontier of $B_{i}$, where $i=1,2, \ldots, m, p=p_{1}$ and $q=p_{m}$. We obtain an initial path $\rho=<p_{1}, \ldots, p_{2}, \ldots, p_{m}>[$ note: it is very likely that there exist further points between $p_{i}$ and $p_{i+1}$ !].

The following steps are modified from Algorithm 1 of [1] (note: only Steps 7.2 and 8.2 are modified!).

4. Let $\varepsilon=10^{10}$ (the chosen accuracy).

5. Compute the length $L_{1}$ of the initial path $\rho$.

6 . Let $q_{1}=p$ and $i=1$.

7. While $i<k-1$ do:

7.1. Let $q_{3}=p_{i}+1$.

7.2. Apply Procedure 4 to compute a point $q_{2}$ on the frontier of $B_{i}$ such that $q_{2}$ is a vertex of an approximate ESP on the Surface of $B_{i-1} \cup B_{i}$ from $q_{i-1}$ to $q_{i+1}$.

7.3. Update $\rho$ by replacing $p_{i}$ by $q_{2}$ [possibly also by some additional points between $p_{i-1}$ and $p_{i}$, and between $p_{i}$ and $p_{i+1}$ !].

7.4. Let $q_{1}=p_{i}$ and $i=i+1$.

8.1. Let $q_{3}=q$.

8.2. Apply Procedure 4 to compute a point $q_{2}$ on the frontier of $B_{m}$ such that $q_{2}$ is a vertex of an approximate ESP on the surface of $B_{m-1} \cup B_{m}$, from $q_{m-1}$ to $q_{m+1}$.

8.3. Update $\rho$ by replacing $p_{k}$ by $q-2$ [note: possibly also by additional points between $p_{m-1}$ and $p_{m}$, and between $p_{m}$ and $\left.p_{m+1} !\right]$.

9. Compute the length $L_{2}$ of the updated path $\rho$.

10. Let $\delta=L_{1}-L_{2}$.

11. If $\delta>\varepsilon$, then let $L_{1}=L_{2}$ and go to Step 7. Otherwise, stop.

We provide a proof of correctness, an analysis of run-time complexity, and an example for this algorithm. It is basically another illustration for the general comments (e.g., in 1410) that the basic idea of rubberband algorithms my be applied efficiently for a diversity of shortest path problems.

\section{Proof of Correctness}

Theorem 1. The approximate path computed by the Main Algorithm is an approximate ESP on the surface of $\Pi$. 
Proof. Let $\left\{B_{1}, B_{2}, \ldots, B_{m}\right\}$ be the step bands computed by Step 2 of the Main Algorithm. Let $\rho_{i}=B_{i} \cap B_{i+1}$, where $i=1,2, \ldots, m-1$. For each point $p_{i} \in \rho_{i}$, where $i=1,2, \ldots, m-1$, the length of the surface path

$$
\rho=<p_{1}, \ldots, p_{2}, \ldots, p_{m}>
$$

is a continuous function defined on $\rho_{1} \cdot \rho_{2} \cdot \ldots \cdot \rho_{m-1}$, denoted by $\Pi_{i=1}^{m-1} \rho_{i}$.

Since each $\rho_{i}$ is a closed set, where $i=1,2, \ldots, m-1, \Pi_{i=1}^{m-1} \rho_{i}$ is a closed set as well.

Since $\rho$ is continuous, for each $\varepsilon>0$ and for each $P=\left(p_{1}, p_{2}, \ldots, p_{m-1}\right) \in$ $\Pi_{i=1}^{m-1} \rho_{i}$, there exists a $\delta_{\varepsilon}>0$, such that for each $P^{\prime} \in U\left(P, \delta_{\varepsilon}\right)$, the difference between the lengths (i.e., of the outputs) of the Main Algorithm by using either $P$ or $P^{\prime}$ as an initial path, is not more than $\varepsilon$.

We can now construct an open cover for $\Pi_{i=1}^{m-1} \rho_{i}$ as follows:

$$
O_{\varepsilon}=\left\{U\left(P, \delta_{\varepsilon}\right): P \in \Pi_{i=1}^{m-1} \rho_{i}\right\}
$$

By the finite cover principle of mathematical analysis, there exists a finite subcover of $O_{\varepsilon}$ which can cover $\Pi_{i=1}^{m-1} \rho_{i}$. This implies that the number of approximate ESPs obtained by the Main Algorithm is finite. In analogy to the proof of Lemma 24 of [14], the number of approximate ESPs obtained by the Main Algorithm is only one. This proves the theorem.

\section{Time Complexity}

This section analyses, step by step, the time complexity for each of the procedures and the Main Algorithm as presented in the previous section.

Lemma 1. Procedure 1 can be computed in time $\mathcal{O}\left(|V(\Pi)|^{2}\right)$.

Proof. In our data structure we identify adjacent vertices for each vertex; so Steps 1 and 4 can be computed in time $\mathcal{O}(|V(\Pi)|)$. Step 2 can be computed in time $\mathcal{O}\left(\left|N_{p}\right|\right)$. Step 3 can be computed in time $\mathcal{O}(1)$. Step 5 can be computed in time $\mathcal{O}\left(\left|N_{v}\right|\right)$. Step 6 can be computed in time $\mathcal{O}(1)$. The loop, from Step 4 to Step 6 , is computed in time $\mathcal{O}\left(|V(\Pi)|^{2}\right)$. Step 7 can be computed in time $\mathcal{O}(|V|)$. Therefore, Procedure 1 can be computed in time $\mathcal{O}\left(|V(\Pi)|^{2}\right)$.

Lemma 2. Procedure 2 can be computed in time $\mathcal{O}\left(\left|\Pi_{1}\right|^{2}\right)$.

Proof. Step 1 can be computed in time $\mathcal{O}(1)$. The test in Step 2 can be computed in time $\mathcal{O}\left(\left|V\left(\rho_{1}\right)\right|\right)$. Step 2.1 can be computed in time $\mathcal{O}\left(\left|V\left(\Pi_{1}\right)\right|\right)$. Step 2.2 can be computed in time $\mathcal{O}\left(\left|V\left(\Pi_{2}\right)\right|\right)$. Step 2.3 can be computed in time $\mathcal{O}\left(\left|V\left(\Pi_{1}\right)\right|\right)$. Steps 2.4 and 2.5 can be computed in time $\mathcal{O}(1)$. The loop, from Step 2 to Step 2.5 , is computed in time $\mathcal{O}\left(\left|\Pi_{1}\right| \cdot\left|V\left(\rho_{1}\right)\right|\right) \leq \mathcal{O}\left(\left|\Pi_{1}\right|^{2}\right)$. Step 3 can be computed in time $\mathcal{O}(|S|)$. Therefore, Procedure 2 can be computed in time $\mathcal{O}\left(\left|\Pi_{1}\right|^{2}\right)$. 
The following Lemma is obvious. Step 1.4 .1 can be computed in time $\mathcal{O}(1)$.

Lemma 3. Procedure 3 can be computed in time $\mathcal{O}\left(\left|V\left(G_{B}\right)\right|\right)$.

Lemma 4. Procedure 4 has time complexity $\kappa_{1} \cdot \mathcal{O}\left(\left|V\left(\rho_{2}\right)\right| \cdot\left|V\left(B_{1} \cup B_{2}\right)\right|\right)$.

Proof. By Lemma 3. Step 1.1 can be computed in time $\mathcal{O}\left(\left|V\left(B_{i}\right)\right|\right)$, where $i=$ 1, 2. By Theorem 1.4 of [11], Step 1.2 has time complexity

$$
\kappa_{1} \cdot \mathcal{O}\left(\left|V\left(\rho_{2}\right)\right| \cdot\left|V\left(B_{1} \cup B_{2}\right)\right|\right)
$$

where $\kappa_{1}=\left(L_{0}-L\right) / \varepsilon, \varepsilon$ is the accuracy, and $L_{0}$ and $L$ are the lengths of the initial and true path, respectively.

Step 1.3 can be computed in time $\mathcal{O}\left(\left|V\left(\rho_{e_{u_{2}}}\right)\right|\right)$. Step 1.4 .1 can be computed in time $\mathcal{O}(1)$. Step 1.4 .2 can be computed in time $\mathcal{O}\left(\left|V\left(B_{1} \cup B_{2}\right)\right|\right)$. Step 1.5 can be computed in time $\mathcal{O}\left(\left|V\left(\rho_{2}\right)\right|\right)$. Step 1.6 can be computed in time $\mathcal{O}(1)$. The loop, from Step 1.1 to 1.6, can be computed in time

$$
\kappa_{1} \cdot \mathcal{O}\left(\left|V\left(\rho_{2}\right)\right| \cdot\left|V\left(B_{1} \cup B_{2}\right)\right|\right)
$$

Step 2 can be computed in time $\mathcal{O}(|V|)$. Therefore, Procedure 2 can be computed in time $\kappa_{1} \cdot \mathcal{O}\left(\left|V\left(\rho_{2}\right)\right| \cdot\left|V\left(B_{1} \cup B_{2}\right)\right|\right)$.

Theorem 2. The Main Algorithm has time complexity $\kappa_{1} \cdot \kappa_{2} \cdot \mathcal{O}\left(\left|V\left(G_{\Pi}\right)\right|^{2}\right)$.

Proof. By Lemma 1. Step 1 can be computed in time $\mathcal{O}\left(\left|V\left(G_{\Pi}\right)\right|^{2}\right)$. According to Lemma 2. Step 2 can be computed in time $\mathcal{O}\left(\left|V\left(G_{\Pi}\right)\right|^{2}\right)$. Step 3 can be computed in time $\mathcal{O}(|V(\rho)|)$. Steps 4, 6, 10 and 11 can be computed in time $\mathcal{O}(1)$. Steps 5 and 9 can be computed in time $\mathcal{O}(|V(\rho)|)$. Steps 7.1, 7.4, 8.1 can be computed in time $\mathcal{O}(1)$. By Lemma 4. Steps 7.2 and 8.2 can be computed in time $\kappa_{1} \cdot \mathcal{O}\left(\left|V\left(\rho_{j 2}\right)\right| \cdot\left|V\left(B_{i-1} \cup B_{i}\right)\right|\right)$, where $j=i, m$. Steps 7.3 and 8.3 can be computed in time $\mathcal{O}(|V(\rho)|)$. The loop, from Step 7 to 11, can be computed in time $\kappa_{1} \cdot \kappa_{2} \cdot \mathcal{O}\left(\left|V\left(G_{\Pi}\right)\right|^{2}\right)$.

Therefore, the Main Algorithm can be computed in time $\kappa_{1} \cdot \kappa_{2} \cdot \mathcal{O}\left(\left|V\left(G_{\Pi}\right)\right|^{2}\right)$.

\section{An Example}

The following example illustrates the steps of the Main Algorithm. Let $\Pi$ be the unit cube in Figure 5 .

Step 1 computes a cut cycle (which may be not uniquely defined) $\rho_{p q}=$ $A B C D A$.

Step 2 computes step bands $S=\left\{B_{1}, B_{2}, B_{3}\right\}$, where $B_{1}=p, B_{2}$ 's frontiers are two cycles $A B C D A$ and $E F G H E$, and $B_{3}=q$.

Step 3 decides that we use $p I J q$ as an initial surface path from $p$ to $q$ (see Figure [5]. 


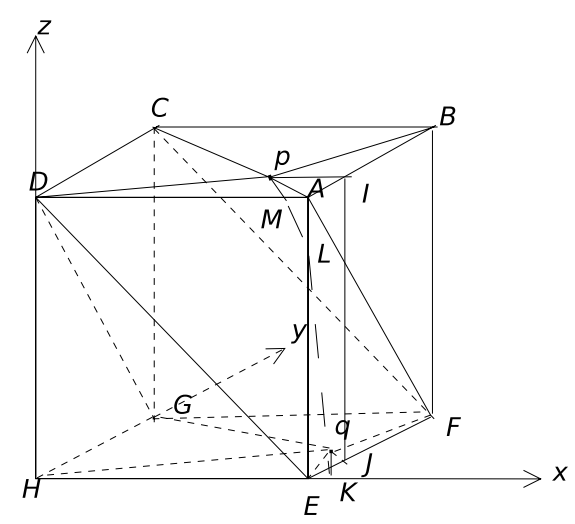

Fig. 5. A unit cube within an $x y z$-coordinate system, where $p=(0.76,0.001,1)$, $q=(0.999,0.001,0) \cdot p I J q$ is an initial surface path from $p$ to $q$ while $p M L K q$ is an approximate surface ESP from $p$ to $q$, where $I \in A B, J, K \in E F, L \in A E$ and $M \in A D$.

In Step 7.2, the algorithm applies Procedure 4 (the initialization procedure of the RBA) and searches each edge of the polygon $A B C D A$; it finds a point $M \in A D$ to update the initial point $I$, and it also inserts a new point $L^{\prime} \in A E$ into the segment between $M$ and $J$.

Analogously, in Step 8.2, the algorithm searches each edge of the polygon $E F G H E$ and finds a point $K \in E F$ for updating the initial point $J$; it also updates point $L^{\prime} \in A E$ by point $L \in A E$ which is between $M$ and $K$.

The algorithm iterates (note: the iteration steps are defined in the Main Algorithm) until the required accuracy is reached.

\section{Conclusions}

The paper presented a rubberband algorithm for computing an approximate surface ESP of a polytope. Although it is not the most efficient, it follows a straightforward design strategy, and is thus easy to implement.

This algorithm generalized an rubberband algorithm designed for solving a 2D ESP of a simple polygon (see [11]) to one which solves the surface ESP of polytopes. This approach is a contribution towards the exploration of efficient approximate algorithms for solving the general ESP problem. This will allow more detailed studies of computer-represented surfaces as typical (e.g.) in biomedical or industrial 3D image analysis.

Acknowledgement. The authors thank the PSIVT reviewers whose comments have been very helpful for revising an earlier version of this paper. 


\section{References}

1. Sharir, M., Schorr, A.: On shortest paths in polyhedral spaces. SIAM J. Computation 15, 193-215 (1986)

2. Reif, J.H., Storer, J.A.: A single-exponential upper bound for shortest paths in three dimensions. J. ACM 41, 1013-1019 (1994)

3. Canny, J., Reif, J.H.: Some algebraic and geometric configurations in pspace, 460467 (1988)

4. Canny, J., Reif, J.: New lower bound techniques for robot motion planning problems, 49-60 (1987)

5. Sharir, M.: On shortest paths amidst convex polyhedra. SIAM J. Computation 16, 561-572 (1987)

6. Gewali, L.P., Ntafos, S., Tollis, I.G.: Path planning in the presence of vertical obstacles. Technical report, Computer Science, University of Texas at Dallas (1989)

7. Mitchell, J.S.B., Mount, D.M., Papadimitriou, C.H.: The discrete geodesic problem. SIAM J. Computation 16, 647-668 (1987)

8. Chen, J., Han, Y.: Shortest paths on a polyhedron, 360-369 (1990)

9. Kapoor, S.: Efficient computation of geodesic shortest paths. In: Proc. ACM Symp. Theory Computation, vol. 1, pp. 770-779 (1999)

10. Li, F., Klette, R.: Rubberband algorithms for solving various $2 \mathrm{~d}$ or $3 \mathrm{~d}$ shortest path problems. In: Proc. Computing: Theory and Applications, Platinum Jubilee Conference of The Indian Statistical Institute, pp. 9-18. IEEE, Los Alamitos (2007)

11. Li, F., Klette, R.: Euclidean shortest paths in simple polygons. Technical report CITR-202, Computer Science Department, The University of Auckland, Auckland (2007), http://www.citr.auckland.ac.nz/techreports/2007/CITR-TR-202.pdf

12. Klette, R., Rosenfeld, A.: Digital Geometry. Morgan Kaufmann, San Francisco (2004)

13. Kiryati, N., Szekely, G.: Estimating shortest paths and minimal distances on digitized three dimensional surfaces. Pattern Recognition 26, 1623-1637 (1993)

14. Li, F., Klette, R.: Exact and approximate algorithms for the calculation of shortest paths. Report 2141, ima, The University of Minnesota, Minneapolis (2006) 